\title{
Congenital Hand Differences: A Review
}

\author{
Yeganeh Ragati-Haghi ${ }^{1}$, Farimah Noorbakhsh ${ }^{1}$ and Amir H. Taghinia ${ }^{1,{ }^{*}}$ \\ ${ }^{1}$ Department of Plastic Surgery, Boston Children's Hospital, Boston, United States \\ "Corresponding author: Assistant Professor of Surgery, Harvard Medical School, Attending Surgeon, Department of Plastic Surgery, Boston Children's Hospital 300 Longwood \\ Ave., Hunnewell, Boston, USA. Tel: +1-6173553421, Email: amir.taghinia@childrens.harvard.edu
}

Received 2017 September 26; Revised 2018 March 04; Accepted 2018 May 15.

\begin{abstract}
This review article will cover common congenital conditions affecting the hand with specific reference to etiology, diagnosis, and treatment. The congenital hand differences that are covered herein are syndactyly, amniotic constriction band, hypoplastic thumb, radial polydactyly, vascular anomalies, and radial longitudinal deficiency. The reader is provided with a brief review of each of these conditions.
\end{abstract}

Keywords: Congenital, Hand, Syndactyly, Polydactyly, Pollicization, Thumb Hypoplasia, Radial Longitudinal Deficiency

\section{Context}

Congenital hand conditions encompass a myriad of differences that affect the hand and upper extremity. The classification and molecular basis of these conditions is constantly updated as new insights into disease processes are elucidated. Nevertheless, the classical defining terms for these conditions remain broadly accepted. Syndactyly refers to the fusion of digits whereas polydactyly refers to the presence of extra digits. Amniotic constriction band is defined as abnormal phenotypic patterns due to fibrotic bands in the amniotic fluid. Hypoplastic thumb refers to deficiencies in different constituents of the thumb. Vascular anomalies include tumors and vascular malformations; the classification of these lesions is undergoing rapid change as new molecular discoveries are made. Radial longitudinal deficiency refers to malformations in the radial axis of the upper extremity. There is enough material on each of these conditions to warrant a separate publication for each. Nevertheless, this publication will provide a comprehensive overview of these conditions and provide references for a deeper perspective if the reader is so inclined.

\section{Genetics and Embryology}

The upper limb buds appear as a bulge on each side of the embryo during the fourth week of gestation. Over the next four weeks, growth and differentiation of these buds occurs to form the human upper extremity. This growth and differentiation is under the influence of multiple signaling centers which dictate the sequential events of development. Hox transcription factors set up a segmental differentiation framework along the proximodistal axis of the limb. Three coordinate axes develop, each via distinctive signaling centers: proximodistal, dorsoventral, and anteroposterior. The ectoderm overlying the distal edge of the limb bud at the dorsoventral boundary forms the apical ectodermal ridge (AER). The formation of this center then initiates the expression of AER-related Wnt and Fgf proteins which act on the underlying mesoderm that, in turn, controls proximal-distal growth of the limb.

A collection of mesodermal cells at the distal ulnar margin is called the zone of polarizing activity (ZPA). These cells control radioulnar patterning and coordinate asymmetric limb mapping. They secrete a potent morphogen called sonic hedgehog (Shh) that induces ulnar proliferation of the limb bud thus expanding its width. It also defines the ulnar four digits of the hand.

By day 33 of development, growth and apoptosis transform the distal portion of the growing limb bud into the hand plate. A complex interaction between the Hox transcription factors and Shh establishes digit number and identity. Apoptosis via discrete interdigital centers leads to isolation of the digits. The joints become evident around the $7^{\text {th }}$ week of gestation, and the limb is fully developed 
by the end of the $8^{\text {th }}$ week.

\section{Classification}

The Oberg-Manske-Tonkin (OMT) $(1,2)$ classification system divides the congenital anomalies of the hand and upper extremity into three main categories: malformations, deformations, and dysplasias. A malformation is the abnormal formation of a part whereas deformation refers to a difference arising after the normal formation of a part. Dysplasia is the abnormality in size, shape, and the organization of cells. Malformations can be further subcategorized based on the axis of development that is predominantly affected (proximal-distal outgrowth, radial-ulnar axis, and dorsal-ventral axis), and also whether the entire limb or only the handplate is involved or an unspecified axis. Malformations include longitudinal deficiency, syndactyly, and polydactyly. Examples of deformations are amniotic constriction band and trigger digit. Dysplasas are further subcategorized into hypertrophy and tumorous conditions, and include macrodactyly and vascular anomalies. Each of the more common congenital hand differences has its own classification system as outlined below.

\section{Syndactyly}

Syn means with or together and dactylos means digit. Syndactyly is the fusion of contiguous digits due to the failure of apoptosis in the apical ectodermal ridge during embryonic development. This abnormality is usually found in infants and the diagnosis is clear. Syndactyly could be simple, only involving the skin or it could be complex, involving bones and other soft tissue compartments. It could be complete or incomplete, depending on the extent of involvement. The incidence of syndactyly is 1 in $2000-3000$ live births. It can be inherited in an autosomal dominant trait with variable expression or reduced penetrance; however, it can also occur sporadically (3). Developmental syndactyly always has a proximal to distal pattern of expression in other words, the proximal web is always affected with variable penetrance distally.

Syndactyly can be associated with other conditions such as Apert or Poland syndrome. In these syndromic conditions, the syndactyly is frequently classified as complicated, which is marked by interposing bony structures between the fingers, making separation more difficult. The timing of syndactyly release varies with surgeon preference and experience, but the most surgeons perform the separation between 12 and 18 months of age. Earlier release should be considered in cases where deviation or deformity is present or impending, such as tight bony distal syndactyly in the $4^{\text {th }}$ webspace. In contrast, in syndromic patients or others with medical comorbidities, operation should be delayed to minimize overall risk of general anesthesia.

The surgeon should pay attention to the webspaces involved, degree of fusion, deviation or angulation, nail involvement, any deficits in range of motion and any family history (syndromes) (Figure 1). Imaging techniques such as plain radiographs are used to ascertain the degree of bone involvement, and guide timing and technique of surgical release. More advanced imaging techniques are usually not necessary. Simultaneous release of adjacent digits is possible, but should be undertaken with the utmost caution to avoid injury to both digital arteries of a single digit. In complex cases, often a digit will have only one digital artery, further risking ischemia if adjacent webs are released simultaneously. The surgeon should also be prepared to do additional soft tissue resurfacing as tissue tension in the digit is easily tolerated on one side, but poorly tolerated on both sides. Although many authors advocate syndactyly release without skin grafts, this is only possible in a subset of cases where the syndactyly is less tightly bound, usually incomplete cases. The authors use skin grafts liberally in almost all cases of syndactyly because the functional outcome appears superior and the downsides of extra time and donor site are quite acceptable. Subsequent immobilization in a long arm cast is critical to ensure good soft tissue healing and predictable skin graft take.

Functional outcome of the surgery depends mostly on the preoperative status of the patient's fingers and hand. In more complicated cases some functional issues such as rotational and angular deformity, and nail deformity are probable.

Complications of syndactyly release include web creep which is the loss of postoperative depth in the webspace. The literature quotes rates of $2 \%$ to $24 \%$ for web creep. In the authors' experience, web creep frequency is low if incisions are placed wisely and skin grafts are used liberally. More immediate complications include partial or complete skin graft loss or flap loss, vascular compromise (in complex and complicated cases), nail deformity, rota- 

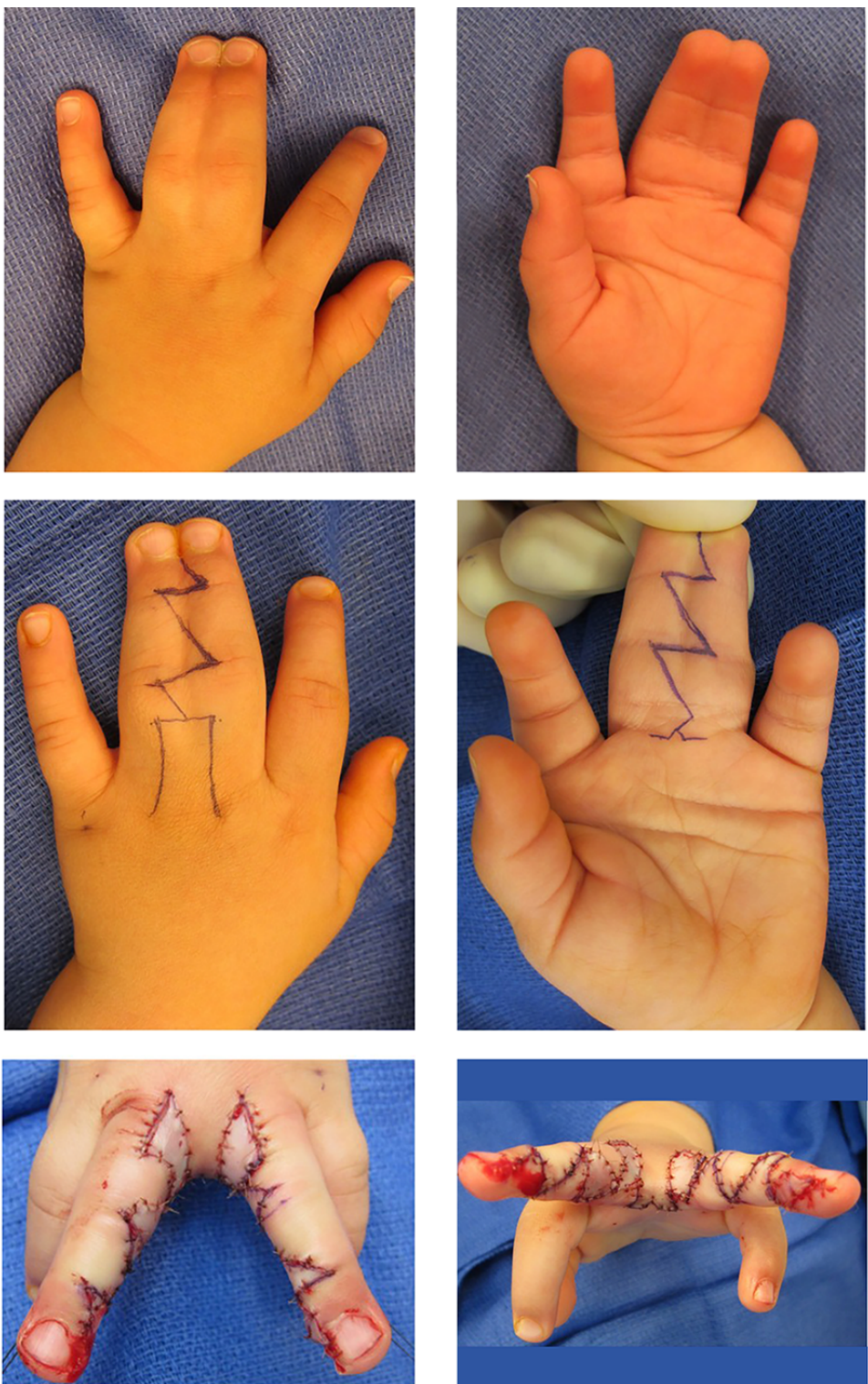

Figure 1. Dorsal (A) and palmar (B) views of a toddler with complete simple syndactyly affecting the third webspace. A dorsal hour-glass flap reconstruction is planned (A) with dorsal and palmar (B) zig zag incisions. The flaps interdigitate, leaving two small open areas proximally which can be covered with full thickness skin grafts from the lower abdomen (E and F).

tional and angular deformities and dense scars. Many of these complications can coexist - for example, poor postoperative immobilization can lead to skin graft loss and subsequent web creep.

\section{Radial Polydactyly}

Polydactyly is the presence of extra digits, and radial polydactyly specifically refers to the presence of extra dig- its involving the thumb ray. It is suggested that an imbalance between the apical ectodermal ridge (AER) and the underlying mesoderm is the cause, but the exact etiology of polydactyly is unknown. The incidence is around 1 in every 3000 live births. Radial polydactyly can be seen in families and there can be associated abnormalities but these are rare (4).

The level of bone and joint involvement aids in establishing the correct diagnosis within the Iowa classifi- 
cation system (5), keeping in mind that soft tissue differences are not included in the classification. Type IV radial polydactyly involves a duplicated proximal phalanx of the thumb, and is the most common type involving approximately $45 \%$ of cases (Figure 2 ). The second most common is type II which is defined as having a duplicated distal phalanx. Additional classification systems have been proposed, but the Iowa system is the most commonly used. In radial polydactyly, the ulnar partner is usually the dominant (better) digit; however, both partners may exhibit findings of thumb hypoplasia and other functional and anatomic abnormalities.

The imaging study of choice in polydactyly is plain radiography. The radiographs demonstrate the exact level and involvement of a polydactyly that may not be possible to diagnose on physical examination.

Small pedunculated ('nubbin') polydactyly can be easily treated in the office if the patient is under a month of age. Local anesthesia is administered and the lesion is either excised with wound closure or ablated with a hemoclip at its base. For older infants or those with widebased polydactyly, the author prefers excision under general anesthesia at the age of 9-12 months. General anesthesia is also required in cases with more extensive involvement. For very complex cases the repair is preferably delayed until the structures are large enough to perform a more technically precise surgery. There are three types of repair of radial polydactyly: resection and reconstruction, the sharing technique, and acral transposition ('ontop plasty'). The choice between these methods is made using the clinical, radiographic, and functional findings. For most cases, resection and reconstruction likely provides the best functional and aesthetic outcome $(6,7)$. Sharing techniques are best for distal cases where the partners are of equal stature and functional status. Acral transposition is reserved for cases where one partner has better distal portions and the other has better proximal portions. This occurs more commonly in proximally involved radial polydactyly such as types V and VI.

\section{Amniotic Constriction Band}

Amniotic constriction band (ACB) is defined as abnormal phenotypic patterns in the limbs or digits allegedly due to fibrotic bands in the amniotic fluid. These phenotypic arrangements could be seen as oligodactyly, acrosyndactyly, lymphedema, nail deformities, joint deviations and stiffness. Amputations may be seen at any level (Figure 3). The incidence of ACB is 1 in 1200 to 15000 live births and it is not familial. It is more common in females than males and more common in African Americans. Various etiologies have been proposed and while each has its strengths and weaknesses, none has been widely accepted. Although multiple different names have also been proposed for this condition, we agree with Rayan's interpretation of nomenclature for this condition (8).

Patterson classified ACB of the limb and/or digit based on level (9):

Type 1: mild digital groove with normal distal extremity,

Type 2: deeper ring with distal deformity, including atrophy or lymphedema,

Type 3: syndactyly with fusion of the distal parts, termed acrosyndactyly or fenestrated syndactyly,

Type 4: amputation distal to the constriction band.

Often called acrosyndactyly or fenestrated syndactyly, digital fusion in ACB is different from developmental syndactyly. In developmental syndactyly, the most proximal part of the webspace is always involved, but in acrosyndactyly the proximal portion is usually uninvolved, manifesting as a small sinus in the most cases. Furthermore, the lengths of digits are usually normal in developmental syndactyly but typically shorter in acrosyndactyly. In cases of severe lymphedema and subsequent ischemia, the band(s) should be released urgently to improve blood flow.

Imaging study of choice is plain radiography though it is not needed in Patterson type I deformity. Doppler ultrasound is considered in evaluating distal blood flow in preoperative planning of deep bands that seem to be fixed to the underlying bone. When surgical planning is considered for separation of complex syndactyly, plain radiography with multiple views is necessary. If there is suspected compression of vessels, nerves, muscles or tendons, then other imaging modality such as MRI is considered, though its utility is questionable in the small patient.

Surgical management is indicated urgently in cases where ischemia and worsening lymphedema are apparent shortly after birth. In other cases, the usual time of surgery is in late infancy or early childhood as there is abundant subcutaneous fat and the skin is highly elastic. Surgery is performed to improve appearance by releasing the band and releasing the attachment of digits. If functional impairment is present, the operation can improve tethering and tendon gliding. In most cases, the main goal is to im- 

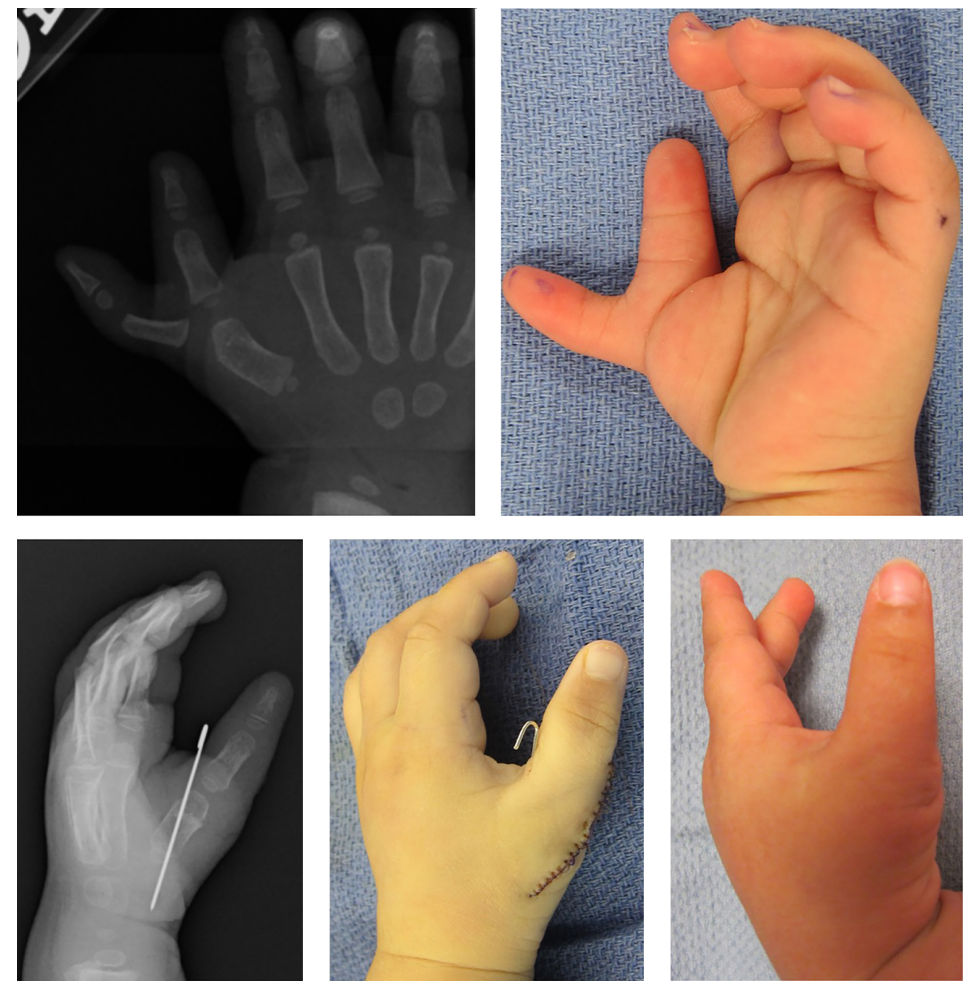

Figure 2. Plain radiograph (A) and clinical images (B) of a patient with Iowa type IV radial polydactyly. The ulnar deviation of the ulnar partner is clear on this palmar view, thus a closing wedge osteotomy is necessary to bring the retained (ulnar) thumb back into neutral alignment. After osteotomy and closure, plain radiograph (C) and clinical photograph (D) show good alignment. One year postoperative view (E) shows excellent alignment and function.
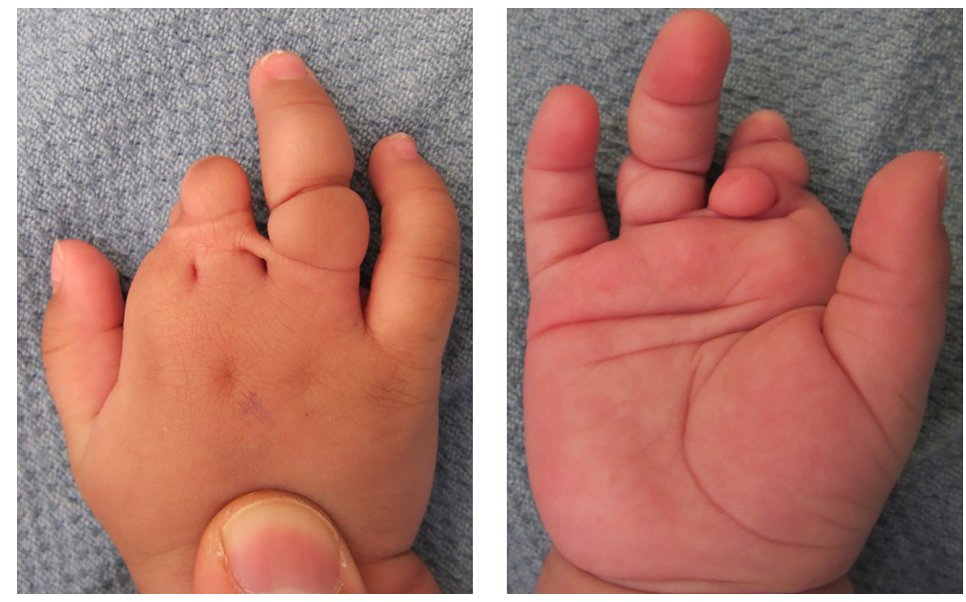

Figure 3. Dorsal (A) and palmar (B) views of a hand with amniotic constriction bands. This hand demonstrates several of the key findings in this condition, including fenestrated syndactyly, amputation, and deep bands.

prove the contour of the limb. It is the authors' preference to avoid Z-plasties especially in deep narrow bands because they are not necessary and introduce additional scarring for no real benefit. In more shallow bands, Z-plasties can be helpful. Staged repair is performed when repairing two adjacent bands to avoid ischemia.

The functional and aesthetic outcome of the surgery is mostly dependent on preoperative status of the bands. 
Adverse outcomes include contour depression or asymmetry, scarring and contracture. Complications of surgery include distal limb ischemia, flap ischemia, wound healing problems, and venous insufficiency.

\section{Hypoplastic Thumb}

The thumb is the functional cornerstone of the hand since it is perfectly adapted for pinching and grasping. Congenital hypoplastic thumb is defined as any condition of the thumb which manifests as deficiency in the bony, articular, musculotendinous or cutaneous components. It can present as a wide spectrum of deficiency from simply a shallow first webspace with a normal thumb to complete absence of the thumb (10).

The thumb is normally broader than the other digits with ample movement in multiple directions, one of the very few features that actually differentiates humans from other animals. The normal first webspace in the hand spans a gentle curvilinear depression between the first and second metacarpophalangeal (MP) joints.

Thumb hypoplasia can be associated with multiple syndromes and/or other environmental causes. The incidence of thumb hypoplasia is 1 out of 100,000 live births, with a large number having associated cardiac anomalies like Holt-Oram syndrome and VACTERL, or hematologic abnormalities like Fanconi anemia and thrombocytopenia absent-radius (TAR). Patients can present any time in life but in mild cases, especially if bilateral or in the non-dominant side, it can go unnoticed until later in life when abnormal appearance or function becomes more pronounced. A practical categorization for thumb hypoplasia which also aids in surgical planning is the modified Blauth classification $(11,12)$.

Type I: thumbs have minimal shortening and narrowing. Surgical intervention is not necessary.

Type II: thumbs have narrowing of the first webspace, hypoplastic intrinsic thenar muscles, and laxity of the metacarpophalangeal(MP) joint. Reconstruction is recommended.

Type IIIA: thumbs have features seen in type II thumbs, but also have extrinsic tendon anomalies and a hypoplastic metacarpal. Reconstruction is recommended.

Type IIIB: thumbs have features seen in type IIIA thumbs, but also have an absent or diminutive proximal metacarpal and instability of the carpometacarpal joint.
Pollicization is the treatment of choice, although a minority of surgeons offer vascularized metatarsophalangeal joint transfer.

Type IV: pouce flottant or floating thumb. The thumb is attached to the hand with a small skin pedicle. It has no function. These thumbs are best treated with pollicization.

Type V: aplasia or complete absence. These thumbs are also best treated with pollicization.

The stability of the MP joint is difficult to assess in an infant because of global joint laxity. However, as the child grows, the laxity becomes more evident on examination. Patients with lax MP joints of the thumb sometimes use a "metacarpal grasp" -using the thumb metacarpal head to brace against an object- thus avoiding the distal thumb because it is unstable.

Intrinsic thenar muscle hypoplasia is usually evident early especially if there is a normal contralateral side. Patients lack strong opposition and there is a hollow or depression in the area of the normal thenar muscles. An incompetent ulnar collateral ligament can sometimes mask a shallow webspace, thus it is best to assess the webspace by the angle achieved between the index and thumb metacarpals. Extrinsic muscle abnormalities include abnormal size, length, strength, and insertion of the thumb flexor and extensors. These anomalies are typically manifested as joint deviations and/or lack of flexion or extension creases. The functional ability of the carpometacarpal joint can sometimes only be made by allowing the child to grow and watching the toddler at play-if the thumb is bypassed in favor of the index finger, the joint is likely nonfunctional.

The first imaging modality used is plain radiography. It can demonstrate the status of the metacarpal, CMC joint, any abnormalities of the index finger, and the status of the distal radius and ulna (to assess radial longitudinal deficiency). MRI can aid in demonstrating soft tissue abnormalities, for example in muscles and tendons, but it is rarely used. The surgeon should focus on treating the individual deficiencies that are present in the thumb, with the modified Blauth classification as a guide.

Index finger pollicization is performed as a procedure transposing the index finger into the position of the thumb, by shortening and repositioning the index finger into a recessed, pronated, and palmarly-abducted position and creating a well-contoured webspace. The authors perform index finger pollicization at around two years of age. The greatest determinant of long term postoperative func- 
tion after pollicization is the preoperative state of the index finger. Abnormal index fingers are typically seen in patients with radial longitudinal deficiency. These neothumbs do not function as well as those who have pollicizations of normal index fingers (13-15).

\section{Vascular Anomalies}

There are two types of vascular anomalies: tumors and vascular malformations. Tumors demonstrate vascular hyperplasia such as hemangiomas while vascular malformations are defined as localized defects of morphogenesis. There are two subcategories for vascular malformations: slow-flow lesions such as capillary, lymphatic, and venous malformations (Figure 4) and fast-flow lesions such as arteriovenous malformations (AVMs). The difference in natural history between hemangiomas and malformations is that hemangiomas have a biphasic growth with both growth and the proliferation early in life and subsequent involution; however, malformations have the tendency to slowly expand throughout life, punctuated by more rapid growth in early life and during puberty. Vascular anomalies can occur anywhere in the upper extremity involving all components of soft tissue, bone, and joint (16).

In regards to pathogenesis of vascular anomalies, there appears to be a role for the somatic activating mutations in the phosphatidyl inositol-3-kinase (PIK3CA)/mammalian target of rapamycin (mTOR) pathway. These (and other) areas are the target of intensive basic science research.

Imaging modalities of choice are ultrasonography as the initial step and MRI for surgical planning. Moreover, angiography is useful for high-flow AVMs. The management of hemangiomas is often conservative with no intervention or with medical therapy such as steroids and beta-blockers. Malformations can undergo interventional radiological treatments including sclerotherapy and embolization. Sclerotherapy around major upper extremity nerves can cause nerve injury; however, there are certain indications for surgical management such as pain, functional compromise, rapid growth, ischemia, and ulceration. Preoperative planning is critical and multiple debulking procedures might be necessary. It is ill-advised to sacrifice normal structures in most of these lesions as complicate eradication is only possible for well-defined, smaller lesions.

The outcome of the vascular malformation surgeries, like most of other hand anomalies, depends on the de- gree of involvement. The more the contractions of joint or tendon/muscle, the lower the chance of improvement after surgery. Bleeding and skin flap compromise are the two most common complications of the surgery in these cases. Poor preoperational planning and incision placement could cause skin flap compromise; however, with meticulous hemostasis and liberal use of drains and hemostatic agents, hematoma and bleeding can be avoided. Unfortunately, recurrence and persistent growth are common problems after surgery. Sclerotherapy should be avoided as a non-operative management in palmar venous malformations. Unless it can be used to fully eradicate a lesion, sclerotherapy (particularly with alcohol) is extremely destructive and can make subsequent surgical resection very difficult and highly risky.

\section{Radial Longitudinal Deficiency}

Radial longitudinal deficiency (RLD) is a congenital anomaly with an incidence of 1 in 30,000 to 100,000 live births. There is hypoplasia of the radial side of the hand, wrist and forearm. Bilateral and unilateral cases happen in equal frequency. There are various degrees of abnormalities in bony, articular, muscular (radial extensors of the wrist), and other soft tissue components such as nerves (radial and or median nerves coalesced into one 'radian' nerve). Developmental anomalies in the apical ectodermal ridge (AER) between the fourth and seventh weeks of gestation are likely responsible.

RLD constitutes a spectrum of anomalies ranging from minimally-shortened radius with a normal hand to complete absence of the radius, radial carpal bone (scaphoid and trapezium) and thumb. Other fingers especially the index finger can be affected but the degree of severity lessens from the radial to ulnar side of the hand. Elbow and shoulder abnormalities can also be present.

RLD is classified based on the degree of involvement. The original Bayne and Klug (17) classification remains widely accepted, though a few modifications have been introduced. The most common types are type III and IV.

Type I: minimal radial shortening of $2 \mathrm{~mm}$ as compared to ulna. The elbow is normal and the thumb is present, though possibly hypoplastic.

Type II: radius is hypoplastic, wrist is deviated, ulna is bowed and thumb and radial carpal bones are absent.

Type III: the radius is partially absent, usually the distal 2/3rd. The thumb and radial carpus are severely affected. A 

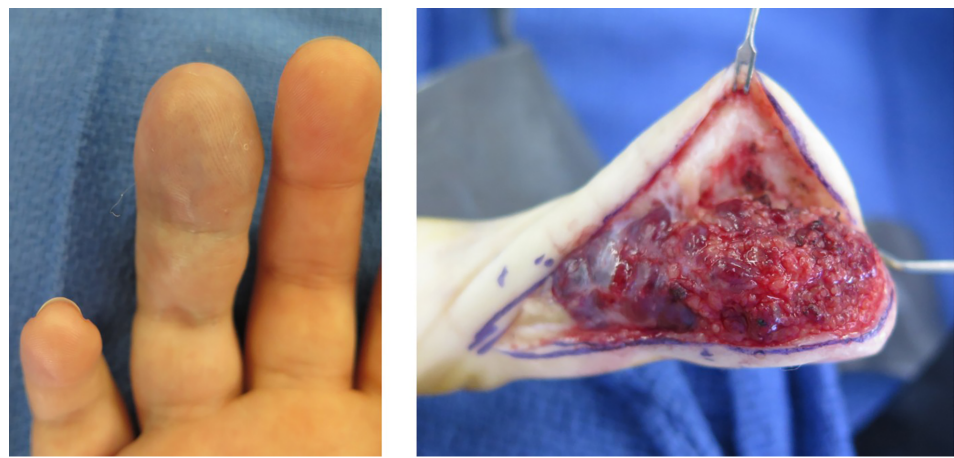

Figure 4. Extensive venous malformation of the ring finger (palmar view, A). A mid-lateral incision has exposed the malformation showing its full extent (B).

fibrous anlage is associated with flexion and pronation of the wrist.

Type IV: the radius is absent and the wrist is severely deviated - sometimes beyond 90 degrees. The thumb is usually absent. Often a small remnant of proximal radius will ossify during the adolescent growth spurt.

Modifications to the original classification system include introduction of three additional types:

1. Type $\mathrm{N}$ : thumb hypoplasia with normal radius.

2. Type 0: absent or hypoplastic carpal bones with normal radius.

3. Type $\mathrm{V}$ : humeral abnormalities.

RLD is frequently associated with other conditions. These conditions can include VACTERL association, HoltOram syndrome, craniofacial microsomia, and Fanconi anemia. The degree of involvement in RLD is detected with plain radiographs which also help in classification. The hand-forearm angle (HFA), and the hand-forearm position (HFP) should be measured on anterioposterior (AP) radiographs. These measurement help assess long-term success of surgical correction versus non-operative treatment. For the majority of patients, non-operative management with splinting and stretching is used early in infancy. This treatment may be a long-term solution for mild RLD (types 0 , I, and II) or a short-term "bridge" solution in preparation to bring the wrist into neutral position for subsequent surgery in more severe cases (types III and IV).

Two methods of surgical management have been introduced for severe cases: centralization and radialization. Centralization involves carving a notch in the carpus to accommodate the distal ulna after bringing it to the central position to correct the deviation. In this operation, the base of the third metacarpal is aligned with the central car- pus and the distal ulna. Tendon transfers are performed to rebalance the wrist muscles. Long-term problems from the original centralization procedure include stiffness and growth arrest. To remedy these issues, Buck-Gramcko introduced radialization which involved first stabilization of the radial carpal column into the distal ulna without carpal resection, and second transfer of the dorso-radial muscle mass ulnarly to contract the deforming forces and provide long-lasting correction. There continues to be debate about what constitutes centralization versus radialization. Preoperative soft tissue distraction techniques have made these operations easier (18). Regardless, long term outcomes show recurrence of wrist radial deviation despite the type of operation undertaken.

\section{References}

1. Oberg KC, Feenstra JM, Manske PR, Tonkin MA. Developmental biology and classification of congenital anomalies of the hand and upper extremity. J Hand Surg Am. 2010;35(12):2066-76. doi: 10.1016/j.jhsa.2010.09.031. [PubMed: 21134615].

2. Tonkin MA, Tolerton SK, Quick TJ, Harvey I, Lawson RD, Smith NC, et al. Classification of congenital anomalies of the hand and upper limb: development and assessment of a new system. $J$ Hand Surg Am. 2013;38(9):1845-53. doi: 10.1016/j.jhsa.2013.03.019. [PubMed: 23684520].

3. McCarroll HR. Congenital anomalies: a 25-year overview. J Hand Surg Am. 2000;25(6):1007-37. doi: 10.1053/jhsu.2000.6457. [PubMed: 11119659].

4. Ezaki M. Radial polydactyly. Hand Clin. 1990;6(4):577-88. [PubMed: 2269673].

5. Wassel HD. The results of surgery for polydactyly of the thumb. A review. Clin Orthop Relat Res. 1969;64:175-93. [PubMed: 4894526].

6. Dijkman RR, van Nieuwenhoven CA, Hovius SE, Hulsemann W. Clinical Presentation, Surgical Treatment, and Outcome in Radial Polydactyly. Handchir Mikrochir Plast Chir. 2016;48(1):10-7. doi: 10.1055/s0042-100460. [PubMed: 26895514]. 
7. Dijkman RR, Selles RW, Hulsemann W, Mann M, Habenicht R, Hovius SE, et al. A Matched Comparative Study of the Bilhaut Procedure Versus Resection and Reconstruction for Treatment of Radial Polydactyly Types II and IV. J Hand Surg Am. 2016;41(5):e73-83. doi: 10.1016/j.jhsa.2015.12.030. [PubMed: 26880498].

8. Rayan GM. Amniotic constriction band. J Hand Surg Am. 2002;27(6):1110-1. [PubMed: 12457366].

9. Patterson TJ. Congenital ring-constrictions. Br J Plast Surg. 1961;14:1-31. [PubMed: 13733379].

10. Chang J, Neligan PC. Plastic Surgery E-Book: Volume 6: Hand and Upper Limb (Expert Consult - Online). 3th ed. Philadelphia: Elsevier Health Sciences; 2012.

11. Blauth W. [The hypoplastic thumb]. Arch Orthop Unfallchir. 1967;62(3):225-46. [PubMed: 4874597].

12. Manske PR, McCarroll HRJr, James M. Type III-A hypoplastic thumb.J Hand Surg Am. 1995;20(2):246-53. doi: 10.1016/S0363-5023(05)80018-8. [PubMed: 7775762].

13. Taghinia AH, Littler JW, Upton J. Refinements in pollicization: a 30-year experience. Plast Reconstr Surg. 2012;130(3):423-33. doi: 10.1097/PRS.ob013e31825dc21f. [PubMed: 22929266].

14. Manske PR, Rotman MB, Dailey LA. Long-term functional results after pollicization for the congenitally deficient thumb. J Hand Surg Am. 1992;17(6):1064-72.

15. Goldfarb CA, Monroe E, Steffen J, Manske PR. Incidence and treatment of complications, suboptimal outcomes, and functional deficiencies after pollicization. J Hand Surg Am. 2009;34(7):1291-7. doi: 10.1016/j.jhsa.2009.04.001. [PubMed: 19540079].

16. Upton J, Taghinia A. Special considerations in vascular anomalies: operative management of upper extremity lesions. Clin Plast Surg. 2011;38(1):143-51. doi:10.1016/j.cps.2010.08.011. [PubMed: 21095479].

17. Bayne LG, Klug MS. Long-term review of the surgical treatment of radial deficiencies. J Hand Surg Am. 1987;12(2):169-79. [PubMed: 3559066].

18. Taghinia AH, Al-Sheikh AA, Upton J. Preoperative soft-tissue distraction for radial longitudinal deficiency: an analysis of indications and outcomes. Plast Reconstr Surg. 2007;120(5):1305-12. 